livraisons

d'Histoire

de l'Architecture

\section{Livraisons de l'histoire de l'architecture}

$30 \mid 2015$

Le dessin d'architecture : œuvre/outil des architectes

?

\title{
Authenticité ou décorativité ? Marques d'imprimeur comme ornements architecturaux
}

Functional or not? Printers' devices as decorative elements of library buildings Authentizität oder Gepränge? Druckerzeichen als architektonisches Ornament

\section{Melinda Simon}

\section{OpenEdition}

Journals

Édition électronique

URL : http://journals.openedition.org/lha/564

DOI : 10.4000//ha.564

ISSN : 1960-5994

\section{Éditeur}

Association Livraisons d'histoire de l'architecture - LHA

\section{Édition imprimée}

Date de publication : 30 décembre 2015

Pagination : 131-147

ISSN : 1627-4970

Référence électronique

Melinda Simon, «Authenticité ou décorativité ? Marques d'imprimeur comme ornements architecturaux », Livraisons de l'histoire de l'architecture [En ligne], 30 | 2015, mis en ligne le 18 décembre 2017, consulté le 11 juin 2020. URL : http://journals.openedition.org//ha/564 ; DOI : https:// doi.org/10.4000//ha.564 
Par Melinda SIMON

\section{AUTHENTICITÉ OU DÉCORATIVITÉ? MARQUES D'IMPRIMEUR COMME ORNEMENTS ARCHITECTURAUX ${ }^{1}$}

Au tournant du $\mathrm{XIX}^{\mathrm{e}}$ et $\mathrm{du} \mathrm{XX}^{\mathrm{e}}$ siècle et pendant les premières décennies du $\mathrm{XX}^{\mathrm{e}}$ siècle, les premières marques d'imprimeur étaient très à la mode. En effet, c'est l'époque de la publication des grandes séries de la littérature relative (par exemple les volumes de Die Büchermarken oder Buchdrucker- und Verlegerzeichen édités par Paul Heintz, suivant une répartition selon les villes, publiés entre 1892-1908, ou les volumes de Die Drucker- und Buchhändlermarken, suivant une répartition selon les unités géographiques, parus entre 1924-1929 à Munich) et de nombreuses monographies importantes (par exemple l'ouvrage de Ronald McKerrow sur les marques d'imprimeur anglais et écossais en $1913^{2}$ ou l'œuvre de base d'Annemarie Meiner en 1922$)^{3}$. En Hongrie, c'est également à cette époque-là que Gyula Végh a fait paraître les marques des libraires de Buda ${ }^{4}$.

Cette vogue est également reflétée dans les revues de l'époque, dont les numéros parus dans les années 1920 et 1930 ont souvent publié - uniquement en tant qu'illustrations, sans études correspondantes - des marques anciennes hongroises ou étrangères. Une revue lancée dans les années 1930 aux États-Unis a choisi comme titre le dauphin d'Alde Manuce (ill. 1), signalant ainsi son intention de s'adresser au public bibliophile ${ }^{5}$.

Même parmi les ornements des bibliothèques américaines construites au cours des décennies en question, les marques typographiques occupent une place domi-

1. Je tiens à remercier l'aide inconditionnelle et professionnelle des collègues bibliothécaires et archivistes américains - sans eux il n'aurait pas été possible de recueillir un corpus méritant d'être analysé : Jesse Lewis (Indiana State Library, Indianapolis); Nathalie Harty (Lithgow Public Library, Augusta); James Stimpert (Johns Hopkins University, Baltimore); Cecile W. Gardner (Boston Public Library); Lois Hendrickson és Tim Johnson (University of Minnesota, Minneapolis) ; Jean E. Meeh Gosebrink és Katherine A. LaBarbera (St. Louis Public Library) ; Arlene Shaner (New York Academy of Medicine) ; Nancy Martin (University of Rochester) ; Elizabeth Chenault (University of North Carolina) ; William Jones (Enoch Pratt Free Library, Baltimore) ; Lisa Moellering (Rice University, Fondren Library, Houston); Janet Olson (Northwestern University Archives, Evanston).

2. Ronald Brunlees McKerrow, Printers' and publishers' devices in England and Scotland 1485-1640, Londres, 1913.

3. Annemarie Meiner, Das Deutsche Signet. Ein Beitrag zur Kulturgeschichte, Leipzig, 1922.

4. Julius von Végh, Ungarische Verleger- und Buchdrucker-Zeichen. 1. Ofner Buchbändlermarken 1488-1525, Budapest, 1923 ; Gyula Végh, The book-marks of the Buda booksellers and publishers 1488-1525, Hungary. A quarterly review of Hungarian life, letters and affairs, 1930, p. 37-41.

5. The Dolphin, a periodical for all people who find pleasure in books, New York (à partir de 1933). 


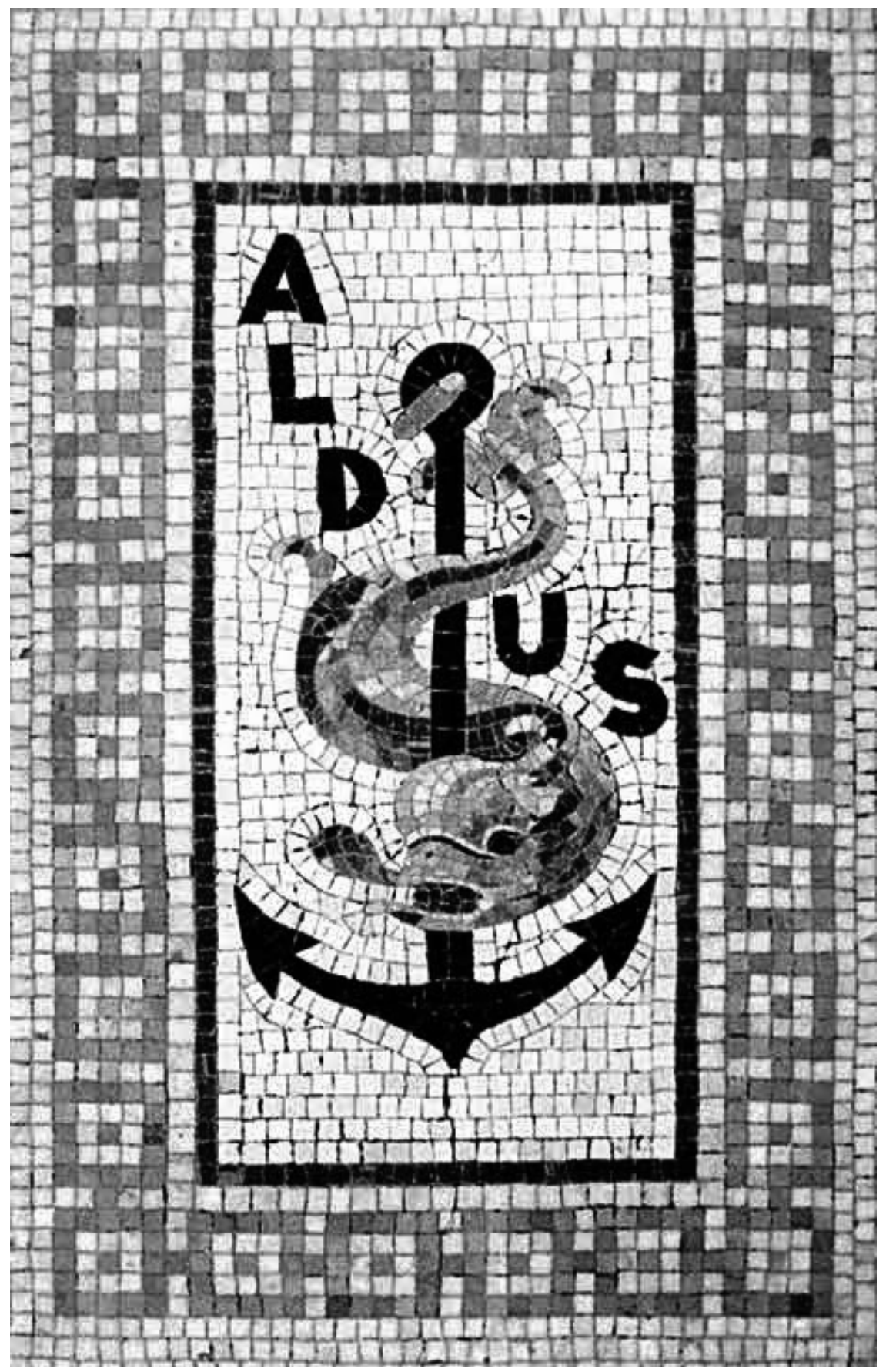

Ill. 1 : Marque typographique d'Aldus Manutius, mosaïque. Madison. (C) Winsconsin Historical Society. 
nante. D'ailleurs, pour l'ornementation des bâtiments, on utilisait fréquemment des figures allégoriques, des statues et reliefs de personnages célèbres ou des citations émanant de ces derniers. À titre d'exemple, Sabine Knopf, dans son étude substantielle $^{6}$, a analysé les figures allégoriques se trouvant sur les façades des palais de libraires et d'éditeurs, construites au tournant du $\mathrm{XIX}^{\mathrm{e}}$ et du $\mathrm{XX}^{\mathrm{e}}$ siècle au centre-ville de Leipzig.

Parmi les bâtiments de bibliothèques faisant l'objet de mes analyses, sur la façade de la Cincinnati University Library on peut trouver entre autres un relief de Johannes Gutenberg. Évidemment, la sculpture ne peut pas être fidèle à la réalité, elle se base uniquement sur les traditions de la représentation de Gutenberg, mais c'est justement grâce à l'image développée par consensus public qu'elle parvient à mettre en évidence l'identité de la personne représentée ${ }^{7}$. Toutefois, sur les ornements architecturaux, on pouvait représenter les bâtiments célèbres d'une ville (par exemple sur les fenêtres de la Lithgow Public Library six bâtiments de la ville d'Augusta) $^{8}$, des armoiries municipales, étatiques ou universitaires (par exemple sur la façade de la Boston Public Library) ${ }^{9}$. Sur trois énormes lustres dorés de la bibliothèque universitaire de Texas, on trouve des citations prises de la Constitution texane $^{10}$ et, dans l'aile orientale de la bibliothèque, une salle a été baptisée Hall of Noble Words à cause des nombreuses citations substantielles marquées sur les poutres en béton du plafond.

À part ces ornements, l'usage massif des marques typographiques semble être en particulier un phénomène typiquement américain. Jusqu'ici, ce sujet n'était traité que superficiellement dans une courte étude de Karen Nipps, qui n'a publié aucune donnée concrète ${ }^{11}$.

Pendant mes recherches, j'ai recueilli des données sur 32 bâtiments de bibliothèques, dont un seul se trouve en Europe (Edinburgh Central Library). Les dates d'inauguration de ces bâtiments sont parlantes : chacun d'eux a été inauguré entre 1890 et 1954 , les dernières étant la bibliothèque de la Rice University de Houston ${ }^{12}$ et de la bibliothèque de la University of Oregon se trouvant dans la ville d'Eugene.

6. Sabine Knopf, "Merkur und Minerva, Greif und Eule, Gutenbergköpfe und Druckerwappen. Schutzpatrone und Insignien von Buchhandel und Buchgewerbe als Bauschmuck ", GutenbergJahrbuch, 2005, p. 245-263.

7. À propos de ce sujet voir George D. Painter, "The True Portrait of Johann Gutenberg ", GutenbergJahrbuch, 1965, p. 73-79 ; George D. Painter, "The Untrue Portraits of Johann Gutenberg ", Gutenberg-Jahrbuch, 1967, p. 54-60.

8. The history of Lithgow Library, URL : http://www.lithgow.lib.me.us

9. Thomas Russell Sullivan, "The New Building of the Boston Public Library ", Scribner's Magazine, $n^{\circ} 1,1896$, p. 83-97.

10. University of Texas, Old Main, URL : http://www.utexas.edu/tours/mainbuilding/oldmain/index. html

11. Karen Nipps, "Printers' devices as decorative elements in library architecture ", The Library Quarterly, n 3, 2013, p. 271-278.

12. Cory Masiak, "On our marks. Symbols of early printers adorn Fondren Reference Room ", The Flyleaf, 1989 , no 40 , p. 2-7. 
Le style des bâtiments présente une gamme variée allant de l'historisme (surtout des réminiscences du style roman et de la renaissance) à l'art déco, ce qui laisse conclure que l'usage des marques typographiques en tant qu'ornement était indépendant du style. Les établissements faisant l'objet de mon étude sont, en premier lieu, des bibliothèques universitaires (18) et, en second lieu, des bibliothèques publiques ou des bibliothèques d'établissements étatiques (14).

Dans presque la moitié des cas, les constructeurs des ornements sont connus : dans la plupart des cas, c'étaient des compagnies basées à New York ou à Boston qui ont produit parfois des milliers d'objets de décoration pour les universités américaines, et ceux qui n'ont pas été utilisés pour un bâtiment donné ont été réinclus dans les plans d'autres bâtiments. L'atelier de G. Owen Bonawit (1891-1971) en est un exemple. Il a fabriqué des vitraux de verre peints notamment pour le compte de l'établissement d'enseignement supérieur Missouri State Teachers College. En 1939, l'atelier a reçu un mandat pour préparer 47 vitraux à l'université (la plupart de ceux-ci représentaient des marques d'imprimeur). Bonawit en a utilisé beaucoup à d'autres endroits, par exemple sur le bâtiment de la Taft School for Boys (Watertown, Connecticut). Ses œuvres se trouvent également en abondance à l'université Yale: d'après une étude, dans la seule Sterling Memorial Library il y en a $887^{13}$.

Le statuaire Domingo Mora (1840-1911) a également procédé de la même manière : il a préparé une cinquantaine de modèles pour ses sculptures représentant des marques d'imprimeur sur la façade de la Boston Public Library mais, plus tard, il n'en a sculpté sur pierre que 33. Les modèles non utilisés pour ledit bâtiment ont été appliqués sur le plafond de la Rhode Island State Library ${ }^{14}$.

Parfois, la fabrication en masse a provoqué des présentations imprécises. Sur l'un des trente reliefs en pierre de la façade de la St. Louis Public Library, on peut même constater une petite erreur: au lieu de la mention "Elsevirs" la mention "Elsivers » y est indiquée. Similairement, dans la mention "Labore et constantia " indiquée sur la marque de Plantin qui comporte un compas, le mot "laborare» apparait erronément sur le vitrail en flint-glass décorant actuellement la collection spéciale de l'université de Chicago, ayant été préparé initialement pour la société d'imprimerie R. R. Donnelley.

Il se peut que parmi les noms des fabricants ceux de Mundhenk et de Schoomaker (New York) ou de Phipps, Slocum and Co. (Boston) ne soient pas très parlants pour nous, mais il y en a un qui est sans doute généralement connu : la compagnie Tiffany de New York. Cette entreprise a fabriqué des vitraux pour la salle de renseignement de la bibliothèque publique de la ville de Winchester (État de Massachusetts) ${ }^{15}$.

13. Missouri State Teachers College, "The dedication of the new library building", November 7 , 1939. Cape Girardeau, 1939. Chapitre intitulé "The college library windows », p. 11-33.

14. Herbert Small, Handbook of the New Public Library in Bosto, Boston, 1895, p. 9.

15. "Art in the library. Tyler memorial windows by Tiffany and Company ", URL: http://www. winpublib.org/about-the-library/art-in-the-library 
Joseph H. Tyler (1829-1892) fut longtemps bibliothécaire de cet établissement, c'est à sa mémoire que sa famille a offert ses vitraux à la bibliothèque en 1894. En ce qui concerne les trois vitraux, celui qui se trouve au milieu représente Gutenberg en train de présenter son invention, la presse typographique et sur les deux vitraux latéraux on peut voir l'arbre du savoir sur les branches duquel les marques des premiers imprimeurs sont suspendues. Parmi toutes les représentations à traiter ci-après, c'est l'une des conceptions les plus originales.

Le nombre des marques représentées est variable : à l'université Harvard, sur le relief se trouvant au-dessus de la porte d'entrée de la bibliothèque Widener ${ }^{16}$, il n'y en a que quatre alors que dans la Library of Congress (Thomas Jefferson Building, Great Hall) on trouve 57 marques au total et que les vitres de la Thompson Memorial Library de Poughkeepsie sont ornées par 82 représentations sur flintglass ${ }^{17}$. Ce sont cependant des cas extrêmes. Si l'on calcule la moyenne des données relatives à tous les établissements, le résultat est de 25 marques par bâtiment (cela peut être considéré comme un chiffre relativement élevé).

En étudiant les techniques utilisées, on peut détecter une diversité fantastique :

- vitres en flint-glass et vitraux peints (12 cas);

- sculptures sur pierre intérieures et extérieures (sur les façades, au-dessus des fenêtres et des portes, sur les jambages, dans les cages d'escaliers, 9 cas) ;

- peintures murales $(6$ cas) ;

- reliefs en bronze au-dessus des portes ou sur les portes (3 cas);

- peintures (sur des plafonds en bois à caissons ou sur les pierres supportant des poutres en béton, 3 cas);

- grilles de bronze placées sur des fenêtres ou des portes $(2$ cas);

- lustres (2 cas);

- sculptures en bois ornant des jambages (1 cas);

- puits d'eau potable, préparé de bronze (1 cas);

- stucs et lames d'or ornant un plafond (1 cas);

- mosaïques de sol (1 cas).

Le choix des marques des premiers imprimeurs (des $\mathrm{XV}^{\mathrm{e}}$ et $\mathrm{XVI}^{\mathrm{e}}$ siècles) est une tendance générale dans l'ornementation mais, à certains endroits, on peut constater la représentation de marques d'éditeur américain moderne (des XIX ${ }^{\mathrm{e}}$ et $\mathrm{XX}^{\mathrm{e}}$ siècles). Dans quelques-unes de ces représentations, les marques modernes n'apparaissent qu'à titre indicatif: Wisconsin Historical Society (1), Boston Public Library (1), University of Cincinnati (2), University of Texas (3), University of Rochester (6), St. Louis Public Library (7), Library of Congress (8). En revanche, on peut constater la séparation intentionnelle et l'application massive de ces marques sur le bâtiment de l'Indiana State Library. Sur le plafond de la History Reference Room, on trouve 21 marques d'imprimeur classiques, alors que le plafond de la

16. Mason Hammond, "A carved tablet showing early printers' marks on the Widener Library", Harvard Library Bulletin, $\mathrm{n}^{\circ} 4,1988$, p. 373-380.

17. A list of the printers' marks in the windows of the Frederick Ferris Thompson Memorial Library, Vassar College. Poughkeepsie, 1917. 
Data Center \& Indiana North Room est orné des marques de 30 éditeurs américains modernes (éditeurs privés et universitaires de grande renommée). Dans les cas où l'imprimeur que l'on souhaitait représenter ne disposait pas de marque, celle-ci a été remplacée soit par les armoiries de la ville où l'imprimeur exerçait son activité, soit par des emblèmes généraux de la sagesse (comme par exemple dans le cas de la St. Louis Public Library) ${ }^{18}$. Les 24 marques modernes figurant parmi les 32 peintures murales de l'Enoch Pratt Free Library (Baltimore) ont également été aménagées de manière à être séparées des marques classiques ${ }^{19}$.

Avant de passer aux marques représentées le plus fréquemment, je tiens à noter que parmi les 32 bibliothèques faisant l'objet de mes analyses, dans trois cas je n'ai pas de données précises concernant les marques représentées (Winchester Public Library, University of Minnesota - Walter Library, Southeast Missouri State University - Kent Library), dans un cas mes données sont incomplètes (Rhode Island State Library). J'ai traité en commun les cas dans lesquels la même marque ou les variantes légèrement différentes de celle-ci ont été utilisées par plusieurs personnes, étant donné que ces marques peuvent être considérées comme identiques du point de vue iconographique. Ainsi, parmi les plus de 150 marques classiques et les marques modernes dont le nombre est approximativement 60, ce sont les marques suivantes qui se trouvent en tête de la liste de popularité.

Marques classiques

\begin{tabular}{|l|c|}
\hline William Caxton, Wynkyn De Worde (Westminster) & 24 \\
\hline Alde Manuce, Paul Manuce (Venise) & 22 \\
\hline Fust et Schöffer (Mainz) & 21 \\
\hline Christophe Plantin (Anvers) & 17 \\
\hline Johannes Frobenius (Bâle) & 12 \\
\hline Robert Estienne, François Estienne (Paris) Noli altum sapere & 11 \\
\hline Lucantonio Giunta, Giunta testvérek (Florence) & 10 \\
\hline Richard Pynson (Londres) & 9 \\
\hline Elzevir (Leiden) Non solus & 9 \\
\hline Simon Vostre (Paris) & 9 \\
\hline Juan Rosembach (Barcelone) & 7 \\
\hline
\end{tabular}

18. The central library building of the Public Library of the city of St. Louis, St. Louis, 1912, p. 15-17.

19. Printers' and publishers' devices shown in the central hall of the new library building, Baltimore, Enoch Pratt Free Library, 1933. 


\begin{tabular}{|l|c|}
\hline Thomas Anshelm (Hagenau) & 6 \\
\hline Richard Grafton (Londres) & 6 \\
\hline Julian Notary (Londres) & 6 \\
\hline Simon de Colines et Guillaume Chaudière (Paris) & 5 \\
\hline Melchior Lotter (Leipzig) & 5 \\
\hline Berthold Rembolt (Paris) & 5 \\
\hline Ottaviano Scotto (Venise) & 5 \\
\hline L'imprimeur de St. Albans & 5 \\
\hline Antoine Vérard (Paris) & 5 \\
\hline Jean Dupré (Lyon) & 4 \\
\hline Johannes Grüninger (Strasbourg) & 4 \\
\hline Jehan Petit (Paris) & 4 \\
\hline Jehan Treschel, Melchior Treschel, Gaspard Treschel (Lyon) & 4 \\
\hline Thomas Vautrollier (Édimbourg et Londres) & 4 \\
\hline Andreas Wechel (Paris) & 4 \\
\hline Nicolaus Jenson (Venise) & 4 \\
\hline Andreas Wechel (Paris) & 4 \\
\hline Nicolaus Jenson (Venise) & 4 \\
\hline
\end{tabular}

\section{Marques modernes}

\begin{tabular}{|l|c|}
\hline Riverside Press (Houghton, Mifflin and Company, Cambridge) & 6 \\
\hline Harper and Brothers (New York) & 4 \\
\hline Bruce Rogers & 4 \\
\hline William Morris & 3 \\
\hline Appleton & 3 \\
\hline Century Co. & 3 \\
\hline De Vinne Press & 3 \\
\hline
\end{tabular}




\begin{tabular}{|l|c|}
\hline Dodd, Mead and Co. & 2 \\
\hline J. B. Lippincott Co. & 2 \\
\hline Benjamin Franklin (Philadelphia) et Christopher Saur (Germantown) & 2 \\
\hline Charles Scribner's Sons & 2 \\
\hline Doubleday Doran & 2 \\
\hline Harvard University Press & 2 \\
\hline Henry Holt \& Co. & 2 \\
\hline Little, Brown \& Co. & 2 \\
\hline Longmans & 2 \\
\hline Yale University Press & 2 \\
\hline
\end{tabular}

Ces chiffres mettent en évidence qu'il y a des cas où le maître d'œuvre - ou l'ingénieur - fait une véritable orgie de marques d'imprimeur et, par conséquent, on peut revoir la même marque à plusieurs endroits, reproduite par différentes techniques; par exemple dans le bâtiment de la Carl Blegen Library de l'Université de Cincinnati on peut remarquer Fust et Schöffer à trois endroits, William Caxton, Alde Manuce ou Christophe Plantin à deux différents endroits.

Ces marques considérées comme importantes se trouvent en général à des endroits remarquables : à titre d'exemple, la marque de William Caxton est placée en milieu de salle, au-dessus de l'horloge, sur la peinture sur bois du caisson du plafond (University of Southern California, Doheny Reading Room), ou bien la marque de Fust et de Schöffer se trouve également en milieu de salle, sur la fenêtre en face de la porte d'entrée (University of Illinois, Main Library).

Compte tenu des critères de sélection, la question se pose de savoir pourquoi ces marques-là se trouvent dans les bibliothèques?

Dans une partie des cas, c'est évidemment la primauté de l'imprimeur qui compte d'un point de vue quelconque : l'importance de Johannes Fust et de Peter Schöffer est due au fait qu'ils étaient les premiers à utiliser des marques typographiques, l'importance de William Caxton est due au fait qu'il était le premier imprimeur à imprimer en langue anglaise, l'imprimeur anonyme du monastère de St. Albans est remarquable parce qu'il était le premier à utiliser une marque typographique en Angleterre, et Alonso de Molina se distingue par l'impression du premier livre sur le continent américain.

Dans d'autres cas, le facteur déterminant est l'importance de l'imprimeur dans l'histoire de l'imprimerie : c'est le cas d'Alde Manuce (Venise), de Christophe Plantin (Anvers), de Johannes Frobenius (Bâle) ou de la famille Elzevier (Leiden et Amsterdam). 
Les marques typographiques témoignant de l'attachement local sont rares et reflètent une attention particulière, comme celles de Walter Chepman et d'Andrew Myllar, les premiers imprimeurs écossais (1473-1533) qui paraissent sur la façade de la bibliothèque municipale d'Édimbourg. Ce même choix conscient se manifeste dans le Andover Hall (Cambridge, Massachusetts) destiné à héberger la Harvard Divinity School et l'Andover Seminary: "Les ornements au plomb de la série de vitres éclairant la tribune d'orgue représentent les marques des premiers imprimeurs allemands, vénitiens et anglais ayant publié des écritures chrétiennes ${ }^{20}$."

Toutefois, ces cas ne représentent qu'une minorité. Malgré le fait que ceux-ci sont représentés le plus fréquemment, ces cas ne constituent pas la majorité des marques typographiques utilisées comme ornements. Alors, dans quoi les personnes compétentes ont-elles puisé ?

On peut considérer que, dans la plupart des cas, les choix sont aléatoires : les marques ont été tout simplement copiées des anciens livres de la bibliothèque ou reproduites sur la base des images publiées dans une monographie spécialisée. La plupart des marques ont été choisies uniquement en raison de leur valeur décorative - à titre d'exemple, on a largement fait usage des marques typographiques sur lesquelles le propriétaire représentait un jeu de mots faisant référence à son propre nom ou de celles qui étaient spectaculaires en elles-mêmes. Le même phénomène peut être constaté dans le cas du choix des marques d'éditeur américain des XIX et $\mathrm{XX}^{\mathrm{e}}$ siècles (voir par exemple le garçonnet de la Riverside Presse jouant de la flûte à bec au bord d'un ruisseau).

Le même effet décoratif pouvait être atteint par la juxtaposition rythmique de marques similaires : comme dans le cas de la construction de la General Library de l'université de Cincinnati, sur la porte de bronze du hall de laquelle on peut remarquer 6 marques combinant la croix, le globe et le chiffre 4 (ce type était très fréquemment utilisé aux $15^{\mathrm{e}}$ et $16^{\mathrm{e}}$ siècles). Il est évident que dans ce cas-là l'identité des imprimeurs n'avait aucune importance ${ }^{21}$. De même, on s'est servi de la rythmicité (mais, cette fois-ci, avec la répétition de la même marque) pour atteindre l'effet décoratif dans le cas des 14 marques peintes en-dessous du plafond de la Doheny Memorial Library de la University of Southern California.

La représentation des marques d'imprimeur est très souvent différente des versions originales (par exemple une marque exposée verticalement a été représentée en exposition horizontale). Dans certains cas, c'est la technique qui a exigé de diverger de la version originale. À titre d'exemple, sur les vitres de la Sperry Room appartenant à la Harvard Divinity School, on a utilisé la forme très simplifiée des marques (même avec des lignes supplémentaires) parce qu'autrement, la vitre en plomb n’aurait pas été stable.

20. "The new buildings of Andover Seminary ", Harvard Graduate's Magazine, 1911, n 78, p. 284-285. (Les citations figurant dans le texte sont des traductions de l'auteur de la présente étude.)

21. Edward A. Henry, A descriptive report on the University of Cincinnati General Library building. With particular attention to the interpretation of the sculpture, the chandeliers, and the printers marks which are used as decoration, Cincinnati, 1951. 
Cependant, dans la plupart des cas, la "falsification » s'explique par des raisons esthétiques et non techniques. En effet, il s'agit du fait que l'architecte aurait voulu voir une décoration dont l'ambiance était entièrement identique à celle du bâtiment rêvé, et à cette fin il a modifié la marque en toute conscience.

L'une des différences correspond à l'utilisation de couleurs : les premières marques typographiques étaient en général des tirages monochromes (noirs), et très rarement des tirages préparées par la méthode de bichromie. Donc, l'utilisation des teintes du gris, du brun et du jaune servait en toute évidence à la création de l'ambiance.

La modification du dessin-même constituait une différence plus importante car, dans ces cas-là, c'est l'authenticité des marques qui a été mise en cause, ce qui fait poser la question suivante : qui avait le droit d'intervenir dans le processus ?

La grande expérience relative des compagnies fabriquant des ornements est évoquée dans le cas de la Harvard Divinty School, où c'est le concepteur, la compagnie Allen \& Collens, qui a proposé une série dont Albert Parker Fitch (doyen de l'Andover Seminary entre 1909-1917) et Owen Gates (bibliothécaire de l'Andover Seminary) ont choisi les marques qui leur plaisaient, et qui ont été ensuite autorisées par William Whittemore (directeur économique de l'Andover Seminary et membre du Comité de construction) et le Comité de construction ${ }^{22}$. Donc, dans ce cas-là, ce n'est pas un spécialiste en histoire de bibliothèques qui a lancé le processus, mais un bibliothécaire qui avait le droit de se prononcer sur la décision.

La situation était relativement simple là où la décision a été prise par une seule personne ou par un groupe restreint (dans la plupart des cas, par le bibliothécaire même ou par une personne qui s'y connaissait en histoire de la culture) : les fresques du plafond de la Indiana State Library ont été choisies par Louis J. Bailey (directeur de la bibliothèque entre 1926 et 1935) ; les vitraux de verre de la Charles Deering Library de la Northwestern University par Theodore Koch, bibliothécaire universitaire $^{23}$; les peintures des vitres de la Lithgow Public Library par les membres du Comité de construction; les sculptures sur pierre de la façade de la Boston Public Library par l'artiste Domingo Mora ; les plans des reliefs de la façade de la St. Louis Public Library par le bibliothécaire (Frederick M. Crunden) et les membres du directoire; les peintures des vitraux de la Southeast Missouri State University vraisemblablement par le réalisateur, G. Owen Bonawit; les grilles de bronze de la porte et les sculptures sur pierre de l'escalier de la University of Rochester par le bibliothécaire (Donald Bean Gilchrist) ; les peintures se trouvant sur les socles de pierre des poutres en béton du Hall of Noble Words à l'université de Texas ont été choisies vraisemblablement par le professeur de philologie classique, William James Battle et par le président du Comité de construction.

La situation était beaucoup plus difficile lorsque l'avis et les points de vue des personnes prenant part au processus de décision étaient nettement divergents. Dans le cas de la bibliothèque de University of Illinois, la situation était la suivante : le

22. Frances O’Donnell, "Glances at artful signs of ages past ", Harvard Divinity Bulletin, 2005, n 1.

23. Janet Olson, Russ Clement, "Storytelling in glass. Deering Library's window medallions ", Footnotes, 2003, no 2, p. 1-2. 
directeur de la bibliothèque (Phineas Windsor) a fait des propositions, les deux architectes (Charles A. Platt et James M. White) en ont choisi et en ont désapprouvé plusieurs, et c'est l'artiste (J. Scott Williams) qui a mené la concertation avec ceux-ci. Heureusement, les notes de ce dernier ont suivi le processus du début jusqu'à la fin. En effet, ces notes ont révélé que le directeur de la bibliothèque a fait une liste de ses propositions à l'architecte principal, en reconnaissant que certaines d'entre elles " ne sont pas artistiques, mais si l'on les omettait, il nous faudrait toujours expliquer les raisons aux chercheurs. J'espère de tout mon cœur que vous et Monsieur Platt intègreront plutôt quelques-unes de celles-ci, et moins de marques des imprimeurs moins connus ${ }^{24}$."

Suite aux affrontements, la plupart des imprimeurs connus ont été inclus dans la sélection, mais les marques de cinq autres (Davidson, Frellon, Wolfe, Berthelet et Hester) ont été également incluses, parce que - selon Williams - "celles-ci ne sont pas tellement importantes, mais elles plaisent à l'architecte. " Dans un autre cas, l'architecte et l'artiste avaient d'abord voulu utiliser la marque typographique de Johannes Amerbach mais, vu que celle-ci n'était pas assez efficace, elle a été remplacée par la marque d'un autre imprimeur de Bâle, celle d'Henri Petri. D'après Williams : "Il n'était pas tellement important qu'Amerbach, mais sa marque esthétique était plus appropriée pour l'objectif visé. " C'est donc une autre illustration du fait que l'argument esthétique l'a emporté sur l'argument professionnel.

L'artiste s'est consumé à mi-chemin, entre les deux positions. Il a déclaré que :

«Évidemment, j’ai consulté des spécialistes en histoire de l'imprimerie concernant le choix des imprimeurs importants, mais cela ne coïncidait pas avec la conception de l'architecte selon laquelle les marques devaient s'intégrer au style architectural et aux dimensions données... J'apprécie chacune des deux positions, celle de l'architecte et celle de l'homme scientifique, et j'essaie de les harmoniser. "

Les architectes ont mis un accent particulier sur le fait que les motifs sur les vitres constituent une unité esthétique avec le style géorgien de la bibliothèque. En février 1926, Williams a écrit à l'architecte principal de l'université :

"L'encadrement orné de feuilles harmonise cette marque d'ailleurs gothique ou à caractère allemand avec les vitres. Platt et son associé, Goldstone ne supportent absolument rien qui puisse avoir un effet baroque à caractère allemand. Néanmoins, mon expert en imprimerie me propose d'utiliser cette marque. »

En effet, il s'agissait de William Caxton, du premier imprimeur anglais qui ne pouvait être négligé en aucun cas, bien que l'architecte « ait refusé l'utilisation complète de la marque du fait de son lourd encadrement carré». Williams a essayé

24. Source de la citation ci-dessus et des citations ultérieures: Amanda M. Flattery, "Printers' marks as library window decorations ", Library Journal, novembre 1927, p. 1015-1017. 
trois différentes possibilités. "Évidemment, c'est le monogramme qui est l'élément important", a-t-il écrit à White. "Pourriez-vous l'accepter avec l'encadrement géorgien?"

Cependant, il connaissait les plus grandes difficultés avec la marque de Johannes Fust et de Peter Schöffer. L'inclusion de celle-ci était importante parce que Gutenberg n'utilisait pas de marque d'imprimeur, mais il y avait des efforts pour le faire représenter d'une certaine manière dans tous les recueils de cette sorte; et la marque Fust-Schöffer en offrait l'occasion. "Je suis fier de cette vitre", a écrit Williams. "Initialement j'aurais voulu placer la marque en haut, mais on me l'a refusé en disant que celle-ci ressemblait à deux reins. Finalement, j'ai peint les portraits de Gutenberg, de Fust et de Schöffer et j’ai mis la marque Fust-Schöffer miniaturisée en bas."

Toutefois, c'est une meilleure solution que celle que l'on peut voir sur les vitraux de la Southeast Missouri State University Kent Library. Là-bas, en dessous de la marque de Josse Bade représentant une presse typographique, l'indication porte le nom de Gutenberg, qui ne se servait pas de marque.

Finalement, l'éloignement abstrait par rapport aux versions originales est illustré par la représentation "secrète " de la marque d'imprimeur de Christophe Plantin sur la sculpture sur pierre de la façade de la Carl Blegen Library (Cincinnati, Ohio). Celle-ci ne représente guère la marque d'imprimeur, elle inclut uniquement une allusion y relative (avec le compas dans la main de l'homme), et de ce fait, cible le visiteur de bibliothèque instruit. L'unique objectif de cette représentation est de l'intégrer harmonieusement parmi les autres panels et de rendre la façade du bâtiment spectaculaire.

T. R. Sullivan, dans un article paru en 1896, un an après l'inauguration de la bibliothèque publique de Boston, exprime son enthousiasme relatif au caractère spectaculaire du nouveau bâtiment de la bibliothèque : "Au-dessus de cet étage on trouve la série des fenêtres ogivales de la salle de lecteurs centrale, entre les arcs des fenêtres il y a une série de médaillons en pierre reproduisant fidèlement les marques de certains imprimeurs célèbres des débuts jusqu'à nos jours. Ces marques typographiques se sont révélées extrêmement décoratives ${ }^{25}$." Toutefois, d'après une autre source " il faut noter que ce n'est pas la réputation d'un imprimeur, mais plutôt l'effet décoratif des marques typographiques qui a motivé leur choix, et en conséquence, de nombreuses personnes relativement insignifiantes ont été incluses ${ }^{26}$. "

L'architecte de la Doheny Memorial Library (University of Southern California), Ralph Adams Cram avait également pour conviction qu'à la conception d'établissements de l'enseignement supérieur le principal but est de susciter le sentiment de dévouement des visiteurs envers la finalité du bâtiment. Ainsi, il a conçu les vitres de

25. Thomas Russell Sullivan, "The new building of the Boston Public Library ", Scribner's Magazine, $\mathrm{n}^{\circ} 1,1896$, p. 83-97, italicisations de l'auteur.

26. Walter Muir Whitehill, "The making of an architectural masterpiece - The Boston Public Library ", American Art Journal, Automne 1970, p. 10. 


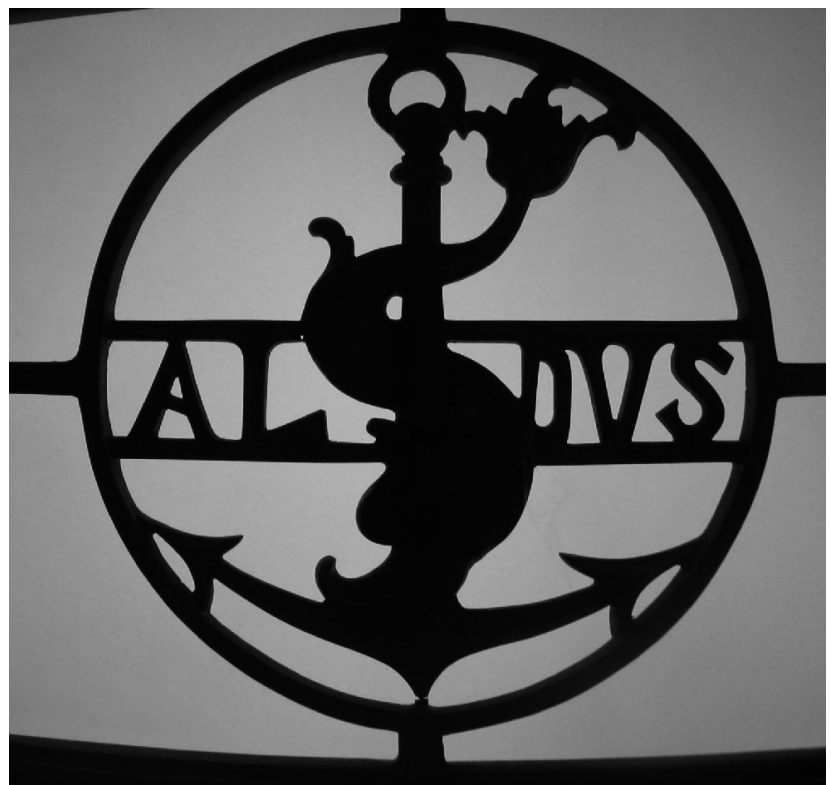

Ill. 2 : Marque typographique d'Aldus Manutius, lustre. New York. CC Cliché Arlene Shaner.

verre, les lustres, les meubles du bâtiment que ceux-ci empruntassent à cet endroit une ambiance semblable à celle des cathédrales ${ }^{27}$.

Pour conclure, je tiens à ajouter que l'utilisation de marques d'imprimeur en tant qu'ornements architecturaux est un phénomène intéressant, bien discernable dans le temps et dans l'espace. Les marques d'imprimeur ont été utilisées uniquement pour décorer les bâtiments de bibliothèques, indépendamment du style des bâtiments, se servant de toutes les techniques possibles. D'après les recherches menées jusqu'à ce jour il s'agit d'une "vogue " répandue (surtout sur la côte orientale des États-Unis), dont la durée estimée englobe la période de 1890 à 1950 . Vu que l'unique objectif était la décorativité et la recherche de l'effet, les marques n'étaient pas toujours représentées de manière réelle, et ont été adaptées aux bâtiments, aux techniques et aux possibilités.

Finalement, on peut conclure que, malgré le fait que les marques d'imprimeur utilisées en tant qu'ornements architecturaux aient perdu leur fonctionnalité originale (et, dans bien des cas, leur forme originale aussi), elles ont obtenu une nouvelle fonctionnalité : elles ont trouvé leur place parmi les motifs décoratifs des bâtiments, quel qu'en soit le style, et contribuent à la création de l'ambiance, même jusqu’à nos jours, et ceci avec beaucoup de succès (ill. 2, ill. 3).

Melinda SIMON

27. University of Southern California - Doheny Memorial Library, URL: http://www.usc.edu/ libraries/locations/doheny/history 


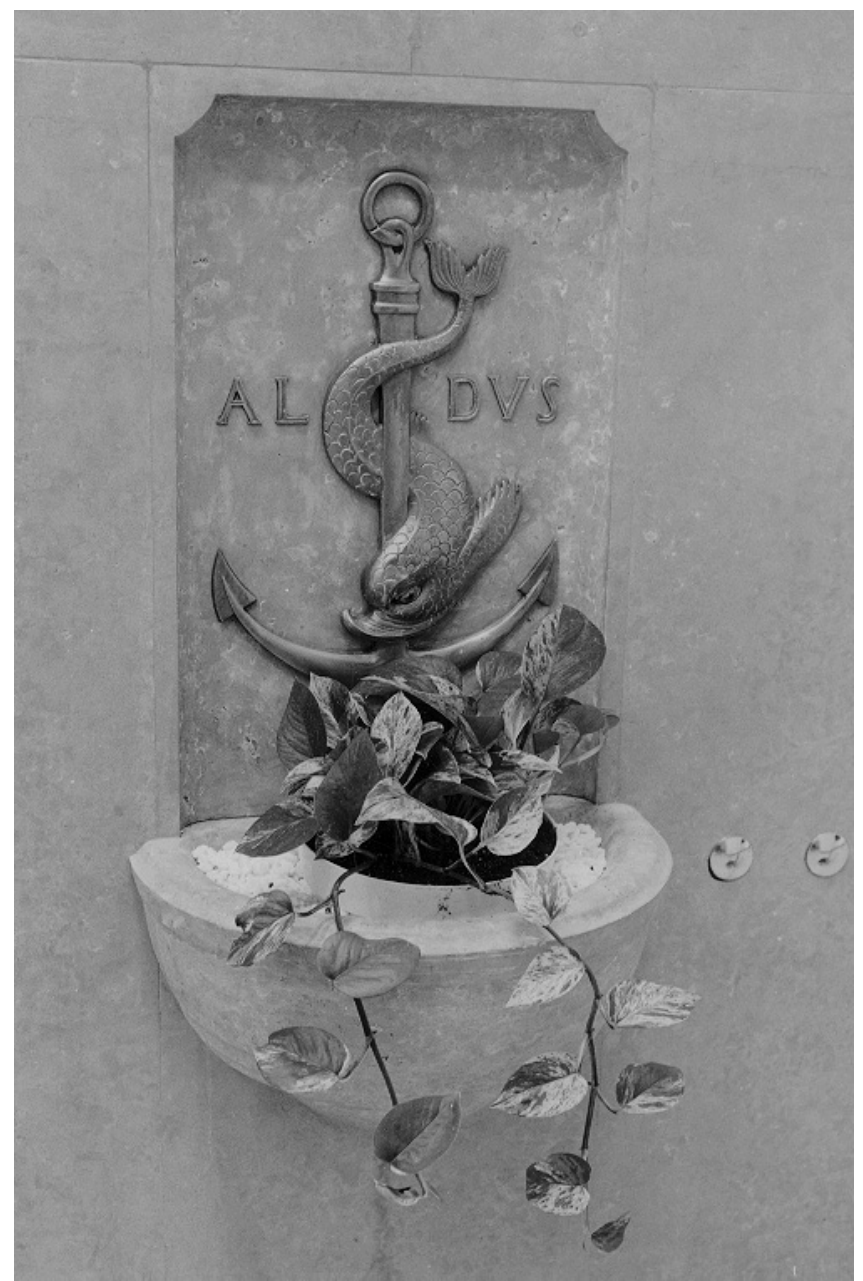

Ill. 3 : Marque typographique d'Aldus Manutius, puits d'eau potable. Cincinnati. (C) University of Cincinnaty.

Livraisons J'bistoire de l'architecture $n^{\circ} 30$ 


\section{Annexe 1 : Données relatives aux bâtiments et aux éléments décoratifs traités dans l'étude}

\begin{tabular}{|c|c|c|c|c|c|}
\hline Établissement & Bâtiment/salle & $\begin{array}{l}\text { Inaugura- } \\
\text { tion du } \\
\text { bâtiment }\end{array}$ & $\begin{array}{l}\text { Nombre } \\
\text { des } \\
\text { marques }\end{array}$ & Technique & Préparateur \\
\hline $\begin{array}{l}\text { Edinburgh Central Library } \\
\text { (Édimbourgh, Écosse, } \\
\text { Grande-Bretagne) }\end{array}$ & & 1890 & 9 & $\begin{array}{l}\text { sculptures sur } \\
\text { pierre placées } \\
\text { sur la façade }\end{array}$ & $\begin{array}{l}\text { McCullogh } \\
\text { (Londres) }\end{array}$ \\
\hline $\begin{array}{l}\text { Morrisson-Reeves Library } \\
\text { (Richmond, Indiana) }\end{array}$ & Reading Room & 1893 & 4 & vitraux peints & $\begin{array}{l}\text { Tiffany \& Co } \\
\text { (New York) }\end{array}$ \\
\hline $\begin{array}{l}\text { Winchester Public Library } \\
\text { (Winchester, Massachusetts) }\end{array}$ & Reference Room & 1894 & inconnu & vitraux peints & $\begin{array}{l}\text { Tiffany \& Co } \\
\text { (New York) }\end{array}$ \\
\hline $\begin{array}{l}\text { Boston Public Library } \\
\text { (Boston, Massachusetts) }\end{array}$ & McKim Building & 1895 & 33 & $\begin{array}{l}\text { sculptures sur } \\
\text { pierre placée } \\
\text { sur la façade }\end{array}$ & Domingo Mora \\
\hline Carnegie Library (Pittsburg) & $\begin{array}{l}\text { Reference Services } \\
\text { Department }\end{array}$ & 1895 & 6 & $\begin{array}{l}\text { peintures } \\
\text { murales }\end{array}$ & \\
\hline $\begin{array}{l}\text { Lithgow Public Library } \\
\text { (Augusta, Maine) }\end{array}$ & Reading Room & 1896 & 32 & vitraux peints & $\begin{array}{l}\text { Phipps, } \\
\text { Slocum and Co. } \\
\text { (Boston) }\end{array}$ \\
\hline $\begin{array}{l}\text { Library of Congress } \\
\text { (Washington D.C.) }\end{array}$ & $\begin{array}{l}\text { Thomas Jefferson } \\
\text { Building, } \\
\text { Great Hall }\end{array}$ & 1897 & 57 & $\begin{array}{l}56 \text { fresques sur } \\
\text { le plafond, } \\
1 \text { relief sur une } \\
\text { porte en bronze }\end{array}$ & \\
\hline $\begin{array}{l}\text { Nichols Memorial Library } \\
\text { (Kingston, New Hampshire) }\end{array}$ & Reading room & 1898 & 11 & $\begin{array}{l}\text { vitres en flint- } \\
\text { glass }\end{array}$ & \\
\hline $\begin{array}{l}\text { Wisconsin Historical Society } \\
\text { (Madison, Wisconsin) }\end{array}$ & $\begin{array}{l}\text { Historical Society } \\
\text { Library }\end{array}$ & 1900 & 6 & $\begin{array}{l}\text { mosaïque de } \\
\text { sol }\end{array}$ & \\
\hline $\begin{array}{l}\text { Rhode Island State House } \\
\text { (Providence, Rhode Island) }\end{array}$ & $\begin{array}{l}\text { Rhode Island State } \\
\text { Library }\end{array}$ & 1904 & 16 & $\begin{array}{l}\text { stucs sur le } \\
\text { plafond, lames } \\
\text { d'or }\end{array}$ & Domingo Mora \\
\hline $\begin{array}{l}\text { Vassar College } \\
\text { (Poughkeepsie, New York) }\end{array}$ & $\begin{array}{l}\text { Frederick Ferris } \\
\text { Thompson } \\
\text { Memorial Library }\end{array}$ & 1905 & 82 & $\begin{array}{l}\text { vitres en flint- } \\
\text { glass }\end{array}$ & $\begin{array}{l}\text { John Hardman } \\
\text { \& Company } \\
\text { (Birmingham) }\end{array}$ \\
\hline $\begin{array}{l}\text { Harvard Divinity School } \\
\text { (Cambridge, Massachusetts) }\end{array}$ & $\begin{array}{l}\text { Andover Hall, } \\
\text { Sperry Room }\end{array}$ & 1911 & 24 & $\begin{array}{l}\text { vitres en flint- } \\
\text { glass }\end{array}$ & \\
\hline $\begin{array}{l}\text { St. Louis Public Library } \\
\text { (St. Louis, Missouri) }\end{array}$ & $\begin{array}{l}\text { Central Library } \\
\text { Building }\end{array}$ & 1912 & 30 & $\begin{array}{l}\text { sculptures sur } \\
\text { pierre placées } \\
\text { sur la façade }\end{array}$ & \\
\hline
\end{tabular}




\begin{tabular}{|c|c|c|c|c|c|}
\hline $\begin{array}{l}\text { Harvard University } \\
\text { (Cambridge, Massachusetts) }\end{array}$ & $\begin{array}{l}\text { Harry Elkins } \\
\text { Widener Memorial } \\
\text { Library }\end{array}$ & 1915 & 4 & $\begin{array}{l}\text { sculptures } \\
\text { surpierre } \\
\text { au-dessus de la } \\
\text { porte d'entrée }\end{array}$ & \\
\hline $\begin{array}{l}\text { University of Illinois } \\
\text { (Urbana-Champaign, } \\
\text { Illinois) }\end{array}$ & Main Library & 1923 & 27 & vitraux peints & $\begin{array}{l}\text { J. Scott } \\
\text { Williams }\end{array}$ \\
\hline $\begin{array}{l}\text { University of Minnesota, } \\
\text { Institute of Technology } \\
\text { (Minneapolis, Minnesota) }\end{array}$ & Walter Library & 1924 & 12 & $\begin{array}{l}\text { sculptures de } \\
\text { pierre et de } \\
\text { bois placées sur } \\
\text { le jambage de } \\
\text { portes intérieures }\end{array}$ & \\
\hline $\begin{array}{l}\text { Iowa State University } \\
\text { (Ames, Iowa) }\end{array}$ & Parks Library & 1925 & 7 & $\begin{array}{l}\text { sculptures sur } \\
\text { pierre }\end{array}$ & \\
\hline $\begin{array}{l}\text { University of California } \\
\text { (Los Angeles, California) }\end{array}$ & University Library & 1927 & 40 & $\begin{array}{l}\text { peintures } \\
\text { murales }\end{array}$ & \\
\hline University of Chicago & $\begin{array}{l}\text { Special Collections } \\
\text { Research Center }\end{array}$ & 1929 & 8 & $\begin{array}{l}\text { vitres en flint- } \\
\text { glass }\end{array}$ & Edgar Miller \\
\hline University of North Carolina & $\begin{array}{l}\text { Wilson Library, } \\
\text { Main Reference } \\
\text { Room }\end{array}$ & 1929 & 4 & lustres & \\
\hline \multirow[t]{5}{*}{$\begin{array}{l}\text { University of Cincinnati } \\
\text { (Cincinnati, Ohio) }\end{array}$} & Carl Blegen Library & 1930 & 28 & $\begin{array}{l}1 \text { puits } \\
\text { d'eau potable }\end{array}$ & \\
\hline & & & & $\begin{array}{l}6 \text { sculptures } \\
\text { sur pierre }\end{array}$ & $\begin{array}{l}\text { Mundhenk } \\
\text { és Schoomaker } \\
\text { (New York) }\end{array}$ \\
\hline & & & & $\begin{array}{l}6 \text { lingots en } \\
\text { bronze placées } \\
\text { sur des portes }\end{array}$ & \\
\hline & & & & $\begin{array}{l}7 \text { grilles de } \\
\text { fenêtre préparées } \\
\text { de bronze }\end{array}$ & \\
\hline & & & & $\begin{array}{l}14 \text { reliefs } \\
\text { en bronze placés } \\
\text { au-dessus de } \\
\text { portes }\end{array}$ & \\
\hline $\begin{array}{l}\text { Johns Hopkins University } \\
\text { (Baltimore, Maryland) }\end{array}$ & $\begin{array}{l}\text { Albert D. Hutzler } \\
\text { University Library, } \\
\text { Gilman Hall }\end{array}$ & 1930 & 19 & vitraux peints & $\begin{array}{l}\text { J. Scott } \\
\text { Williams }\end{array}$ \\
\hline
\end{tabular}




\begin{tabular}{|c|c|c|c|c|c|}
\hline $\begin{array}{l}\text { University of Rochester } \\
\text { (Rochester, New York) }\end{array}$ & Rush Rhees Library & 1930 & 41 & $\begin{array}{l}16 \text { grilles en } \\
\text { bronze placées } \\
\text { sur des portes, } \\
25 \text { sculptures } \\
\text { sur pierre dans } \\
\text { des cages } \\
\text { d'escaliers }\end{array}$ & $\begin{array}{l}\text { Philipp Merz } \\
\text { (Gordon and } \\
\text { Kaelber) }\end{array}$ \\
\hline $\begin{array}{l}\text { University of Southern } \\
\text { California (Los Angeles, } \\
\text { California) }\end{array}$ & $\begin{array}{l}\text { Doheny Memorial } \\
\text { Library, Los Angeles } \\
\text { Times Reference } \\
\text { Room }\end{array}$ & 1931 & 14 & $\begin{array}{l}\text { peintures sur } \\
\text { bois réalisées } \\
\text { sur des plafonds } \\
\text { à caissons }\end{array}$ & $\begin{array}{l}\text { John } \\
\text { D. Smeraldi }\end{array}$ \\
\hline $\begin{array}{l}\text { Enoch Pratt Free Library } \\
\text { (Baltimore, Maryland) }\end{array}$ & Central Hall & 1931 & 32 & $\begin{array}{l}\text { peintures } \\
\text { murales }\end{array}$ & \\
\hline $\begin{array}{l}\text { Northwestern University } \\
\text { Library (Evanston, Illinois) }\end{array}$ & $\begin{array}{l}\text { Charles Deering } \\
\text { Library, Seminar } \\
\text { Room }\end{array}$ & 1932 & 7 & vitraux peints & $\begin{array}{l}\text { G. Owen } \\
\text { Bonawit }\end{array}$ \\
\hline $\begin{array}{l}\text { Indiana State Library } \\
\text { (Indianapolis, Indiana) }\end{array}$ & $\begin{array}{l}\text { Data Center \& } \\
\text { Indiana North Room }\end{array}$ & 1934 & 51 & $\begin{array}{l}\text { fresques sur le } \\
\text { plafond }\end{array}$ & \\
\hline $\begin{array}{l}\text { The New York Academy } \\
\text { of Medicine (New York) }\end{array}$ & $\begin{array}{l}\text { Library, Malloch } \\
\text { Rare Book Room }\end{array}$ & 1934 & 9 & lustres & \\
\hline $\begin{array}{l}\text { University of Texas } \\
\text { (Austin, Texas) }\end{array}$ & $\begin{array}{l}\text { Main Building, Life } \\
\text { Science Library, } \\
\text { Hall of Noble Words }\end{array}$ & 1937 & 16 & $\begin{array}{l}\text { peintures sur } \\
\text { les pierres } \\
\text { supportant des } \\
\text { poutres en béton }\end{array}$ & \\
\hline $\begin{array}{l}\text { Southeast Missouri } \\
\text { State University } \\
\text { (Cape Girardeau, Missouri) }\end{array}$ & Kent Library & 1939 & 47 & vitraux peints & $\begin{array}{l}\text { G. Owen } \\
\text { Bonawit }\end{array}$ \\
\hline $\begin{array}{l}\text { University of Houston } \\
\text { (Texas) }\end{array}$ & $\begin{array}{l}\text { Rice University } \\
\text { Library, Fondren } \\
\text { Reference Room }\end{array}$ & 1949 & 8 & $\begin{array}{l}\text { peintures } \\
\text { murales }\end{array}$ & \\
\hline $\begin{array}{l}\text { University of Oregon } \\
\text { (Eugene, Oregon) }\end{array}$ & Allen Hall & 1954 & 9 & $\begin{array}{l}\text { sculptures sur } \\
\text { pierre }\end{array}$ & \\
\hline
\end{tabular}


